Original Research Paper

\title{
Chilled Water VAV System Optimization and Modeling Using Artificial Neural Networks
}

\author{
${ }^{1}$ Rand Talib, ${ }^{1}$ Nabil Nassif, ${ }^{2}$ Maya Arida and ${ }^{2}$ Taher Abu-Lebdeh \\ ${ }^{I}$ Department of Civil and Architectural Engineering and Construction Management, \\ University of Cincinnati, Cincinnati, OH, USA \\ ${ }^{2}$ Department of Civil, Architectural and Environmental Engineering, \\ North Carolina A\&T State University, Greensboro, USA
}

Article history

Received: 01-01-2018

Revised: $18-10-2018$

Accepted: 22-10-2018

Corresponding Author:

Nabil Nassif

Department of Civil and

Architectural Engineering and

Construction Management,

University of Cincinnati,

Cincinnati, OH, USA

Email: nassifnl@uc.edu

\begin{abstract}
It was estimated that about $40 \%$ of total U.S. energy consumption was consumed by the residential and commercial sectors. According to EIA, in 2009, the energy consumption in U.S. homes was $48 \%$ which was down from $58 \%$ in 1993 Residential Energy Consumption Survey (RECS). The development of building energy savings methods and models becomes apparently more necessary for a sustainable future. The cooling coil is an essential component of HVAC systems. The accurate prediction of a cooling coil performance is important in many energy solution applications. This paper discusses the modeling methodologies of a chilled water cooling system using artificial neural networks. The objective of this research paper is to properly develop the model to predict the cooling coil performance accurately. This study utilized data from an existing building located in North Carolina, USA. Data such as chilled water supply temperature, airflow rate, mixture and supply air temperatures and humidity ratios, etc., are collected over the course of three months for developing and testing the model. Multiple neural network structures are tested along with multiple input and output delays to determine the one yielding the optimal results. Moreover, an optimization technique is developed to select premier model that can predict results accurately validated by the actual data. The observations from this research validates the use of artificial neural network model as an accurate tool for predicting the performance of a chilled water air handling unit.
\end{abstract}

Keywords: Building Energy Model, Neural Network, AHU, Fan Coil, GA

\section{Introduction}

Heating, ventilation and air conditioning systems are widely used in buildings to provide occupants with conditioned air and acceptable indoor air quality. As energy concerns continue to grow, the need for more energy efficient systems has increased. The design of these systems constitutes a large impact on the energy usage and operating cost of buildings they serve. Buildings stand for a substantial part of the total energy consumption in the Unites States (Pérez-Lombard et al., 2008). The increase demand on energy savings and cost reductions necessities the development of intelligent energy-saving techniques. The ability of the models to predict performance of such systems is essential to design more energy efficient and sustainable building systems. Moreover, if current energy use continues to grow buildings will become the largest consumer of global energy by 2025 (EIA, 2015). In efforts to reduce the amount of energy consumed by buildings, several energy efficient strategies have been investigated (ASHRAE, 2015; 2016). The chilled water system is one Heating, ventilation and air conditioning systems are widely used in buildings to provide occupants with conditioned air and acceptable indoor air quality. The chilled water system is one of the most commonly used HVAC systems in the both commercial and industrial buildings. These systems are currently used to provide thermal comfort for a wide array of building types, sizes and in different climates. Also, today the majority 
of commercial buildings are equipped with Building Automation Systems (BAS), which have the ability to record and collect large amounts of data (Nassif, 2014). However, even with this modern technology, the majority of structures still do not operate optimally due to a lack of artificial intelligent computational techniques. Thus, the association of the artificial neural networks will be an important method in predicting the performance and designing the heating, ventilation and air conditioning systems (Gallant, 1993). These systems that are inspired by the biological neural network of the brain are becoming one of the next top modeling tools in the industry of computational sience. ANNs and like the human brain learn by experience. In that respect, these models can be used on virtually any individual part to a system as well. So long as a model can be trained by receiving some sample data and a teaching mechanism, it would be possible to adopt this method in predicting the performance of condensers, blowers, boilers, chillers and other components, too. one of the benefits of these computational modeling tools is that its ability to help in the process of designing a less energy consuming building in general but more specifically HVAC systems (Nassif, 2014). Therefore, the first objective of this study is to produce an accurate ANN models capable of predicting the system performance of a chilled water system and secondly this paper proposes this modeling approach be employed by BASs to guarantee human comfort, improved system controls and lower energy consumptions. The optimization method genetic algorithm GA (Goldberg, 1989) developed in this study will determine the optimal time delay and order needed to produce a best ANN structure yielding minimum errors between the simulated and actual data.

\section{Methodology}

In order to conduct the proposed study, the methodology as shown in Fig. 1 is used:

- Data collection: Collecting energy data from an existing building, the hourly energy data is collected on a five-minute interval for three months. These data are used to calibrate the model for accuracy

- Develop a modeling technique: Using artificial neural network methods as a modeling technique to generate a model that will accurately predict the performance of the AHU. Different neural network structures are tested along with various input and feedback delays to determine the one yielding the best results in term of mean square error (MSE) or coefficient of variance
- Develop an optimization technique: A genetic algorithm method was used and integrated into the ANN models to automate the process of finding the best model structure

- Implementation: The proposed method was tested on an existing building. The model performance and optimization techniques are evaluated.

\section{Preprocessing and Data Analysis}

\section{Data Collection}

This research is based on the data collected from an existing building located on the campus of North Carolina A\&T State University, Greensboro, NC. This three-story, eighty-eight thousand square foot structure is a multi-use classroom building conditioned by typical VAV systems. The mechanical system for this building consists of six Air Handling Units (AHUs) and chilled water central plant with two chillers. The arrangement for each AHU, as depicted in Fig. 2, includes a supply and return fan, exhaust, return and outside air dampers and heating and cooling coils.

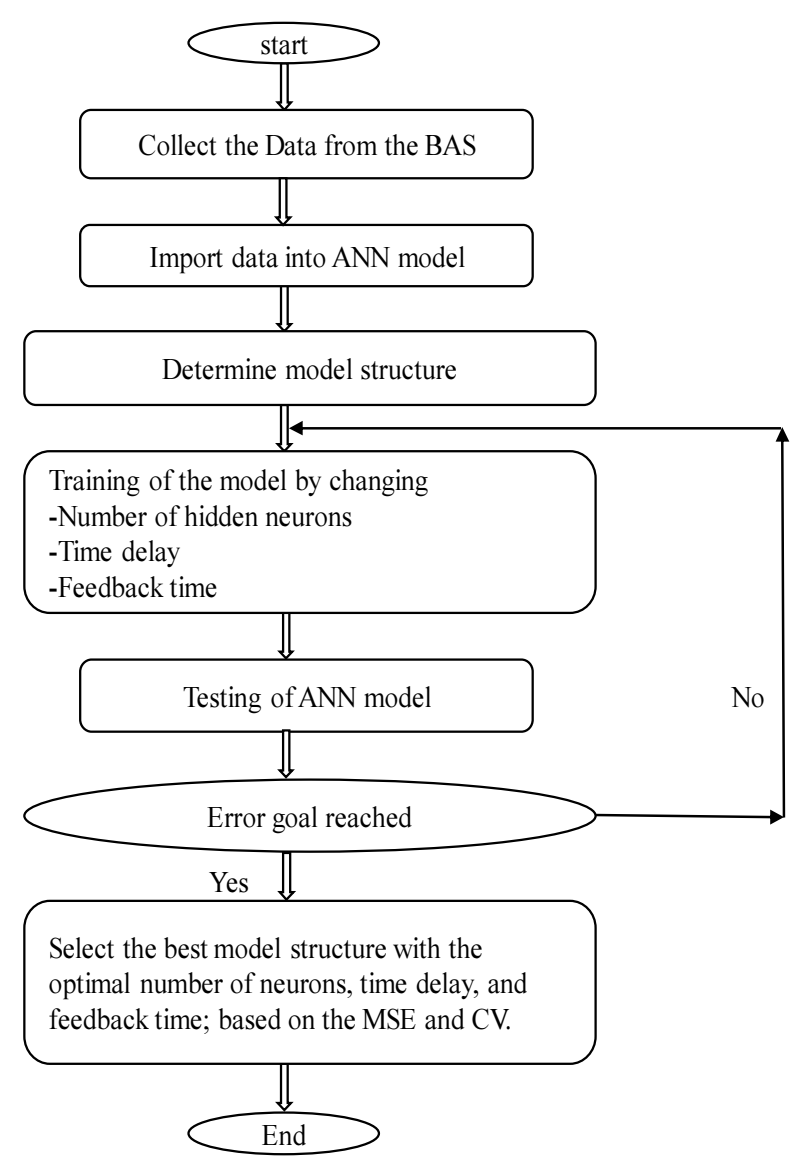

Fig. 1: Workflow diagram of the experiment methodology 


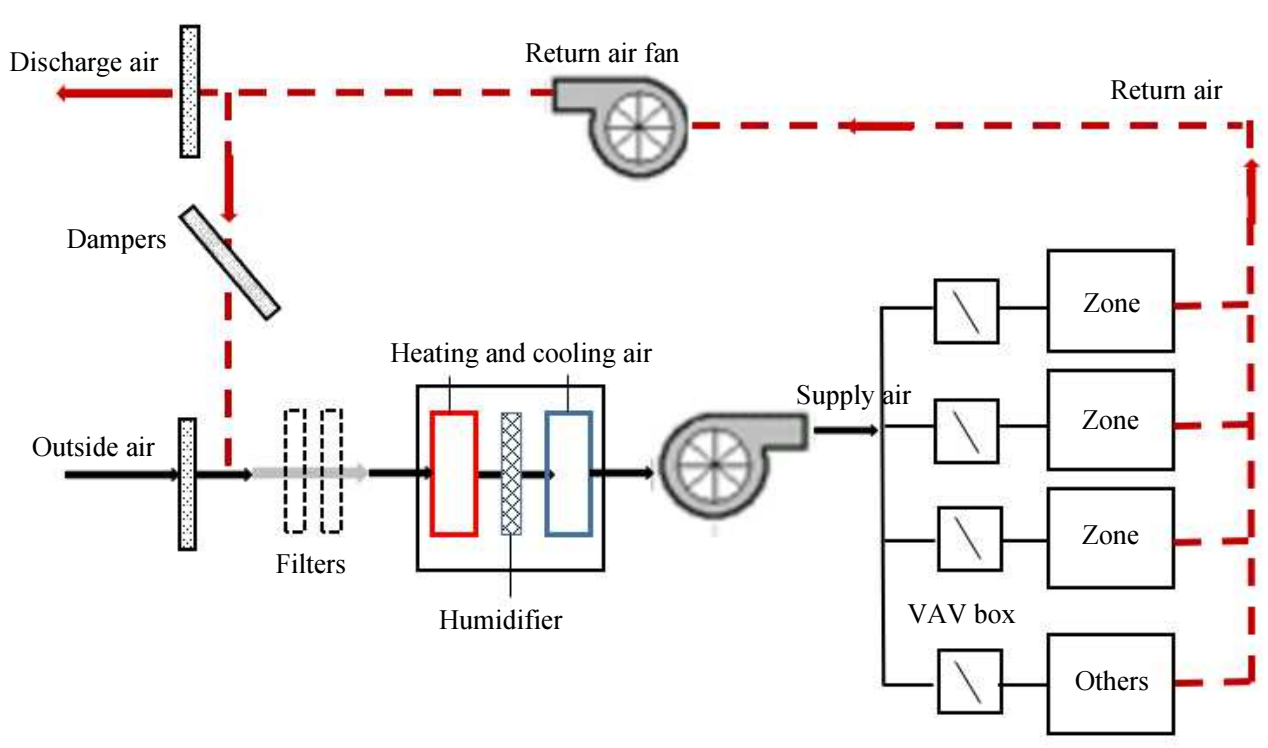

Fig. 2: A schematic of the components inside of a CHW AHU

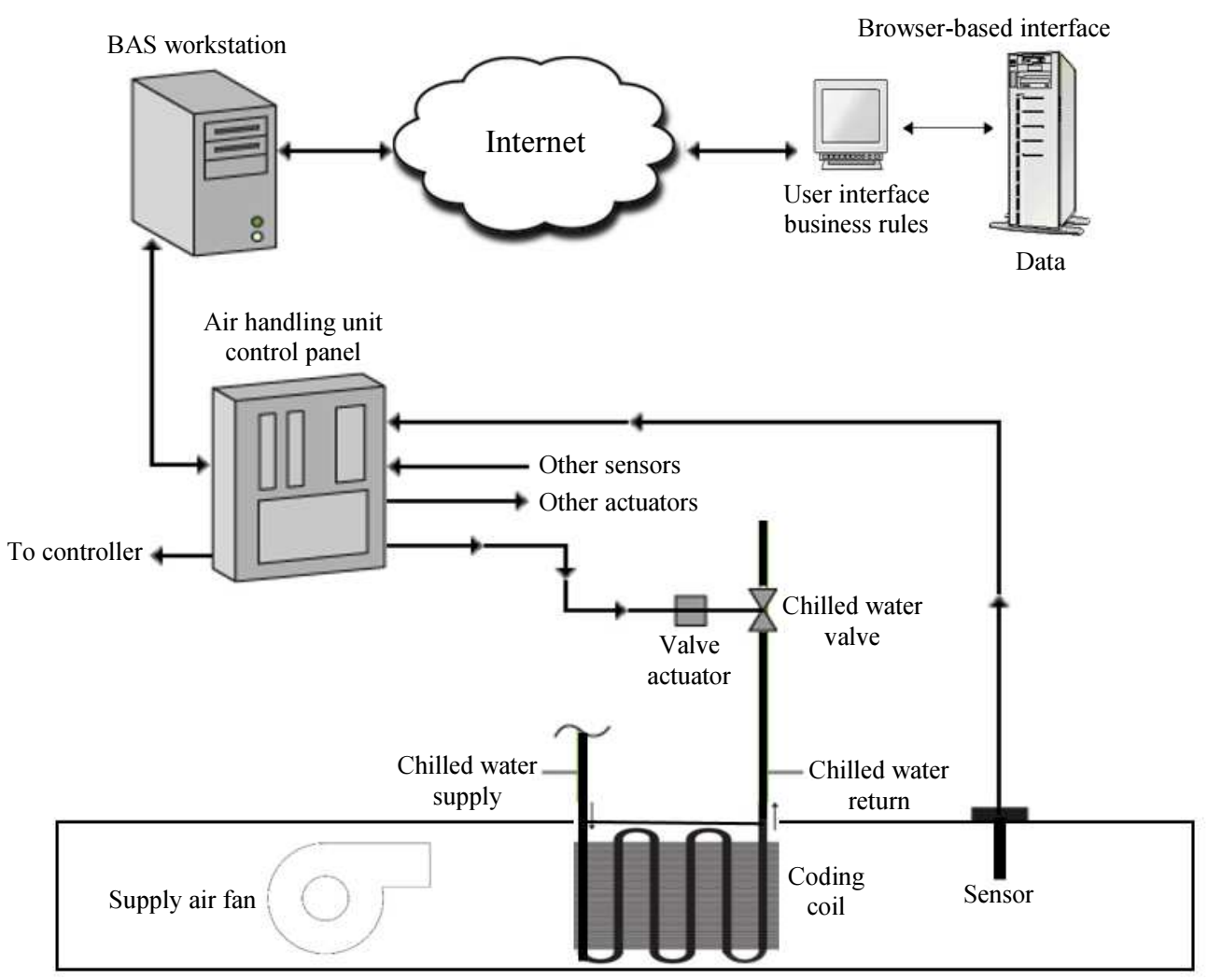

Fig. 3: Building Automation System (BAS) componen

The CHW system investigated is controlled by an online-accessible Building Automation System (BAS) North Carolina's A\&T State University BAS is equipped with BACnet, which is a data communication protocol for building automation and controls established by ASHRAE Standard 135-2008. A Building Automation
System (BAS) consists of sensors, controllers, actuators and software. An operator interfaces with the system via a central workstation or web browser (shown in Fig. 3).

The performance data of the CHW system was trended at five-minute time intervals from November 2014 to February 2015. A total sample points of 28,767 
were collected from the system, representing more than $1500 \mathrm{~h}$ of system run time (system runs about $16 \mathrm{~h}$ per day). The data recorded from the $\mathrm{CHW}$ system covers a temperature range as high as $65^{\circ} \mathrm{F}$ and as low as $50^{\circ} \mathrm{F}$ for the supply air temperature. The building's SAT has a design set point of $55^{\circ} \mathrm{F}$. It is important to note that the BAS software continuously records measurements, even if the CHW system is turned off. These points are removed, because they are repetitive and won't improve the learning capabilities of the ANN models. The collected data are split into two samples designated for training and testing. This task is performed because it is desired that the developed models generate results that are reproducible. The actual data that are generated from a real building are divided into two sets of data (1) is the training set, from November 1 st to December 31 st and (2) testing set was conducted from January 1 st to February 1 st.

\section{Modeling}

The model that is proposed in this research is being computed using Artificial Neural Networks that are computational tools inspired by the central nervous systems of the brain. This tool is used to generate a function based on multiple known inputs (Bishop, 1995).

For this case study, the development and assessment of two data-based models of (1) the chilled water cooling coil and (2) the fan of the selected AHU is explored. These two components are chosen; due to the numerous optimization applications, these components can provide building operators.

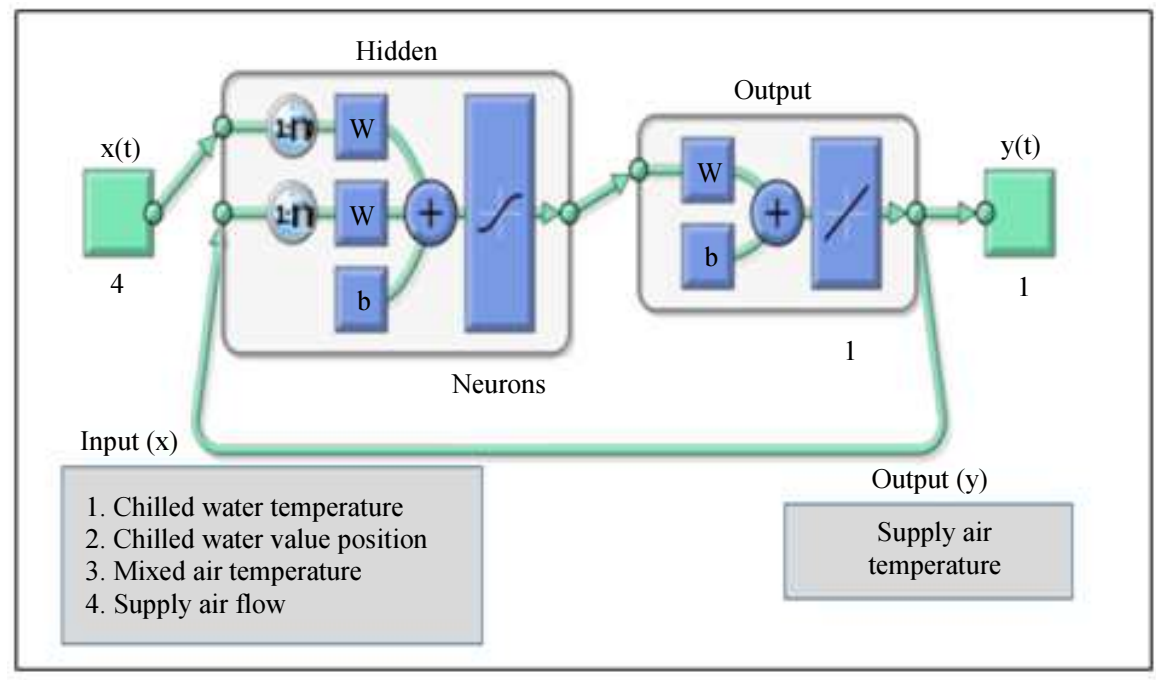

Fig. 4: Displays the inputs/output selected for the cooling coil models

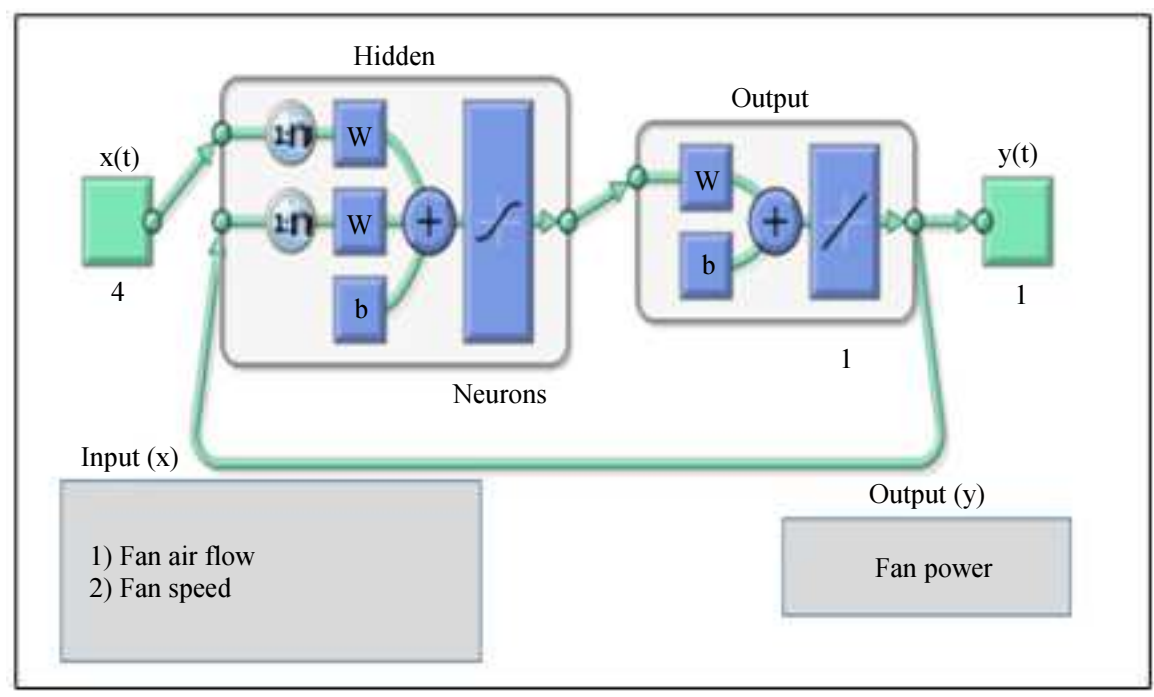

Fig. 5: Displays the inputs/output selected for the supply air fan models 
After the data were collected from the network outputs, statistical model techniques were implemented to interpret the results which are the Mean Square Error (MSE) and the percent Coefficient of Variation or CV\%.

In the case of the chilled water cooling coil model, the supply air temperature is the target output as shown if Fig. 4, while the (1) chilled water temperature, (2) chilled water valve position, (3) mixed air temperature and (4) supply air flow. The supply air temperature is the general measure of performance of a CHW cooling coil and the input variables selected are the greatest contributors to the output variable's performance.

While for the supply fan model, the fan power is selected as a function of the supply air flow and fan speed as show in Fig. 5.

Utilizing the artificial neural network process, various model structures along with different time delays and orders, are investigated to determine the best structure yielding satisfactory accuracy in terms of Mean Square Errors (MSE), root Mean Square Errors (RMSE) and Coefficient of Variances (CV\%). The MSE and CV\% are employed to evaluate the approximating capability of the ANN models. The selected model structures are tested for optimality by using an exhaustive parameter combination. After each model is tested, the results are then evaluated to determine the most optimal model structure and architecture. The models produced from the parametric study will also be evaluated and validated by performing an optimization on the testing data set.

\section{Optimization}

The optimization process includes selecting the best model structure with the least error value in terms of MSE, RMSE and or CV\%. The Genetic algorithm was implemented as a method to select the optimal model that held the lowest error between the actual performance data and the simulated data that were generated throughout the testing period. Moreover, the process variables are (1) input time delays, (2) feedback time delays and (3) number of neurons (hidden layer size). While the objective of it is to find the lowest mean square error MSE, root mean square error RMSE and or coefficient of variance $\mathrm{CV}$. To prevent the process from being extremely complicated a limit was specified. The number of hidden layers of neurons was chosen to be a minimum of 1 and a maximum of a 100. Figure 6 shows the constructed process of optimizing the best model structure for this study. The equations for MSE and CV\% are included.

A Genetic Algorithm (GA) is a search method that is used in the artificial intelligence and computing. is based on the natural selection theory which will choose the best individual (the most fitted) from a population. They are believed to have the ability to find the most fitted individual to the most complex problem because they are capable of working with constrained and unconstrained problems.
There are five steps to the general GA process:

- Initial population

- Fitness function

- Selection

- Crossover

- Mutation

The process of optimization using GA for this research is shown in Fig. 6. The process starts with the main sample size of data. The performance of each individual in the population is evaluated based on the specified constrains. The next step is then using the steps selection, crossover and mutation to choose the stronger variable that will outrun the weaker ones. The process will then be repeated until the maximum number of generation is achieved or an optimal solution is determined. The objective of this process is to find the structure with the lowest error value. The results from this process will then be compared against the results generated through the parametric study to validate the results and the accuracy of the optimization process.

\section{Implementing and Results}

A parametric study was firstly conducted to better understand the performance of the air handling unit, the relationship between the inputs and the outputs with the model that was built is the parameters that will validate the results. Then, the optimization technique is then applied to find the best model structure. The actual data that are generated from a real building are divided into two sets of data (1) is the training set, from November 1 st to December 31 st and (2) testing set was conducted from January 1st to February 1st.

To predict the best model structure that will predict the most accurate results three factors have been changed to predict the best structure of the model those factors are, (1) input time delay intervals of 1-3, (2) feedback time delay intervals of 1-3 as well, (3) while the number of neurons were ranging from 1-100. Each model is then estimated using a developed MATLAB script and evaluated for its predicted performance by conducting the MSE and CV\% for each structure for both training and testing periods as shown in Fig. 7 and 8.

From the charts below, it was observed that few data gave abnormal results in the testing period, those data can be considered an outlier and another simulation was run to get the accurate results. To compensate for possible outlier, additional tests were performed with each network configuration, but between 1 to 5 neurons. After the second simulation was done the data were smoothed out and are in order. The least CV\% and MSE for the testing and training data set is 1.22 and 0.02 respectively. As a result, the model for the testing and training data set with a time delay of 3 , feedback delay of 3 and 45 number of hidden neurons holds the best result for the minimal mean square. 


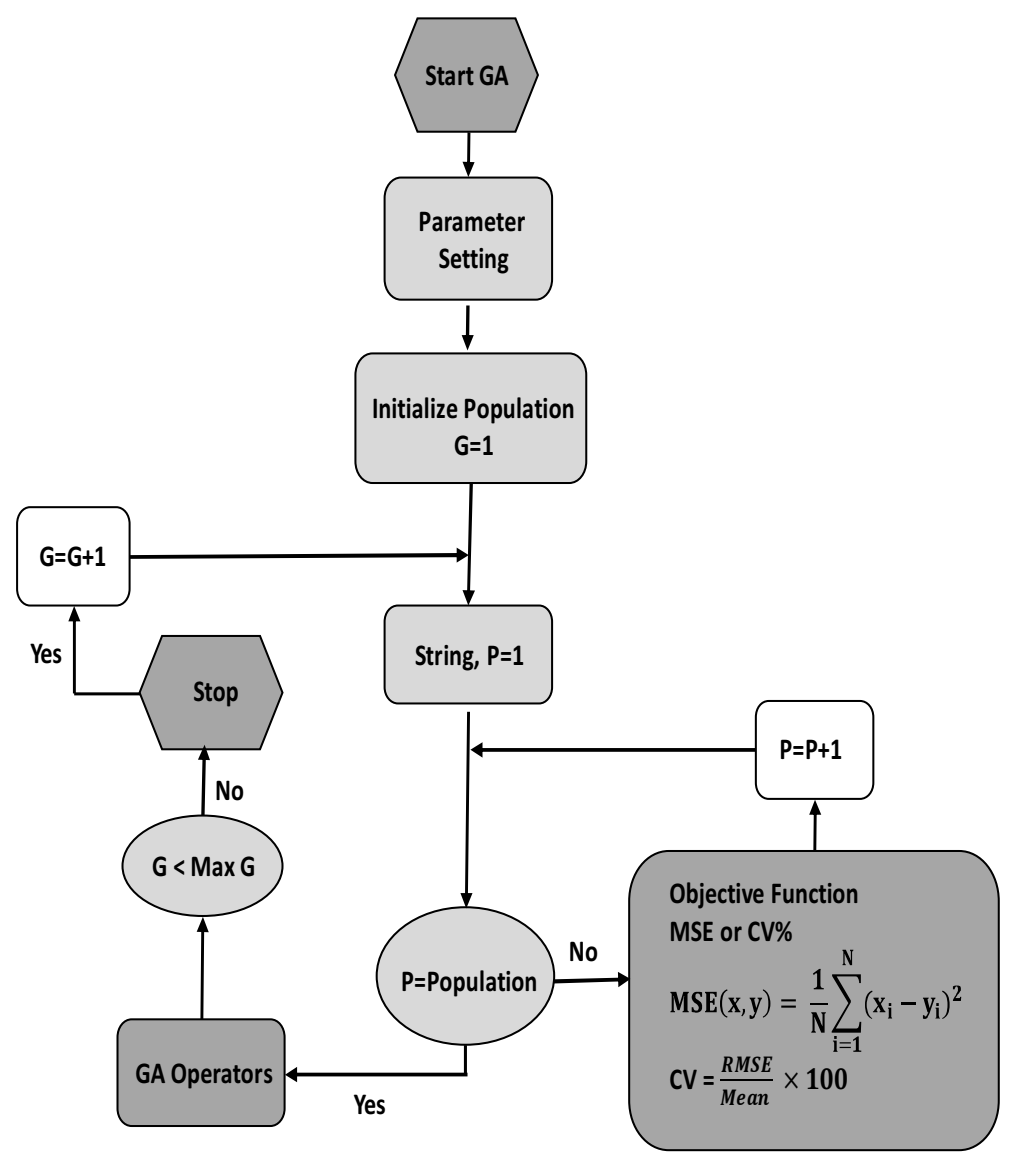

Fig. 6: General layout of the optimization process using GA

Results of the cooling coil model in the texting period

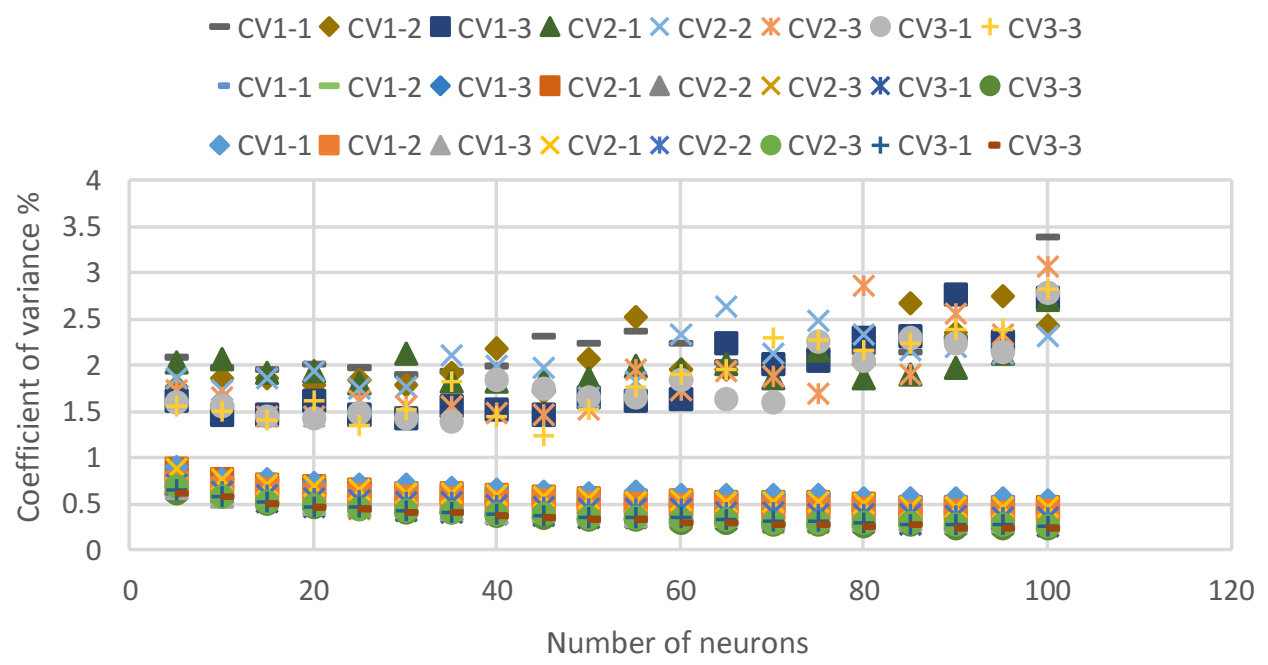

Fig. 7: Resulted CVs\% for testing data set with various time delays and model orders. (CV1-2 means a time delay of 1 and feedback delay of 2) 


\section{Results of the cooling coil model in the training period}

$\rightarrow$ CV1-1 CV1-2 $\triangle$ CV1-3 x CV2-1 *CV2-2 o CV2-3 + CV3-1 -CV3-3

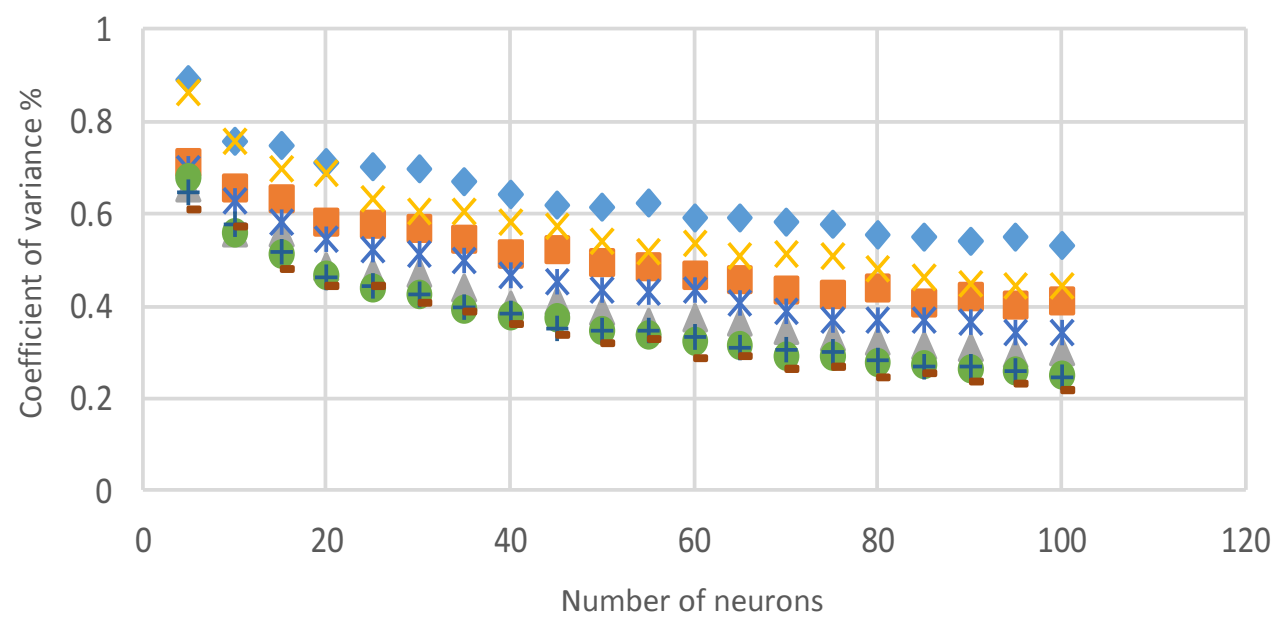

Fig. 8: Resulted CVs\% training data set with various time delays and model orders. (CV1-2 means a time delay of 1 and feedback delay of 2)

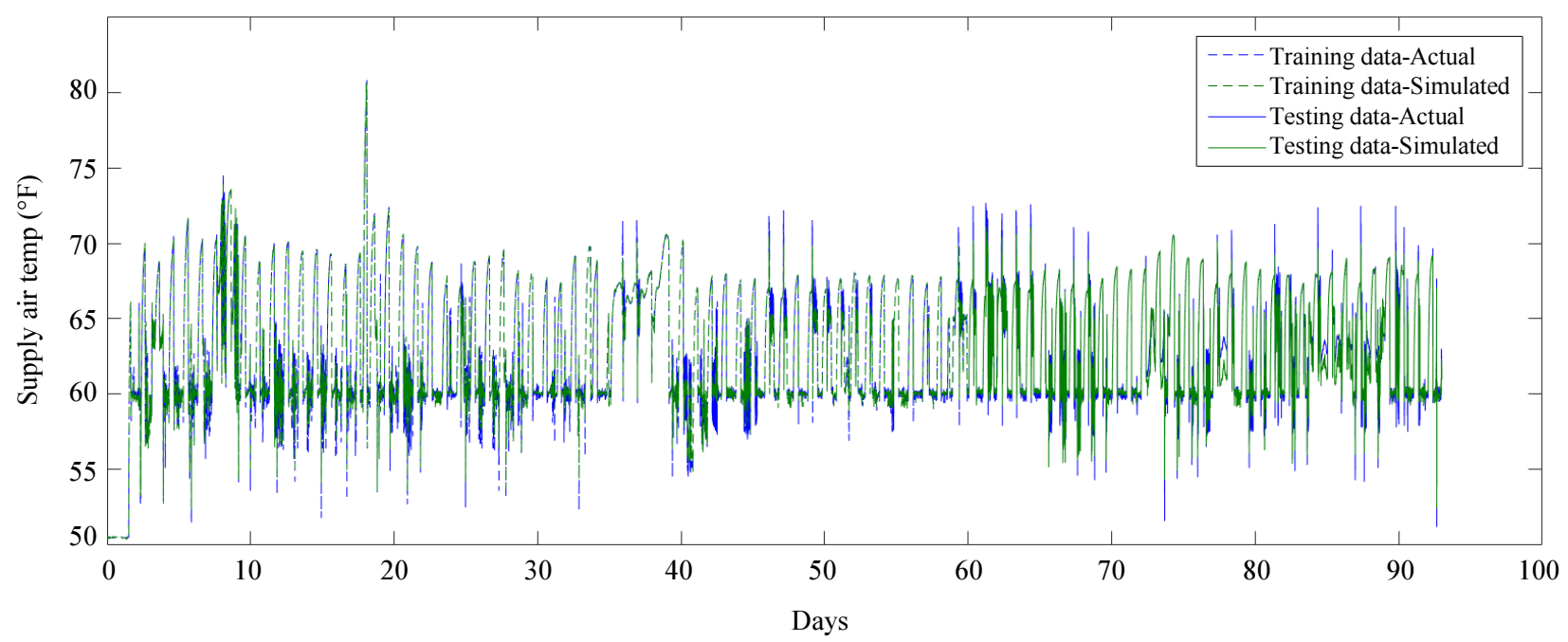

Fig. 9: The simulated and actual data of the cooling coil's performance

Figure 9 shows the simulated and actual data of the cooling coil's performance using best structure of ANN model. The data for training and testing periods are depicted. The optimal model structure that held the lowest error values is indicated using the optimization process as showing in Fig. 6. To check the validity of the optimized model it was compared against the one conducted through the parametric study that was previously explained. It was concluded that the optimal solution that is chosen by the optimization process is similar to the one obtained by the parametric studies, which are illustrated in Fig. 10 and 11. The model with a time delay of 3 , feedback delay of 3 and 45 number of hidden neurons holds the best result having CV\% of 1.2223 and MSE of 0.0173 . 


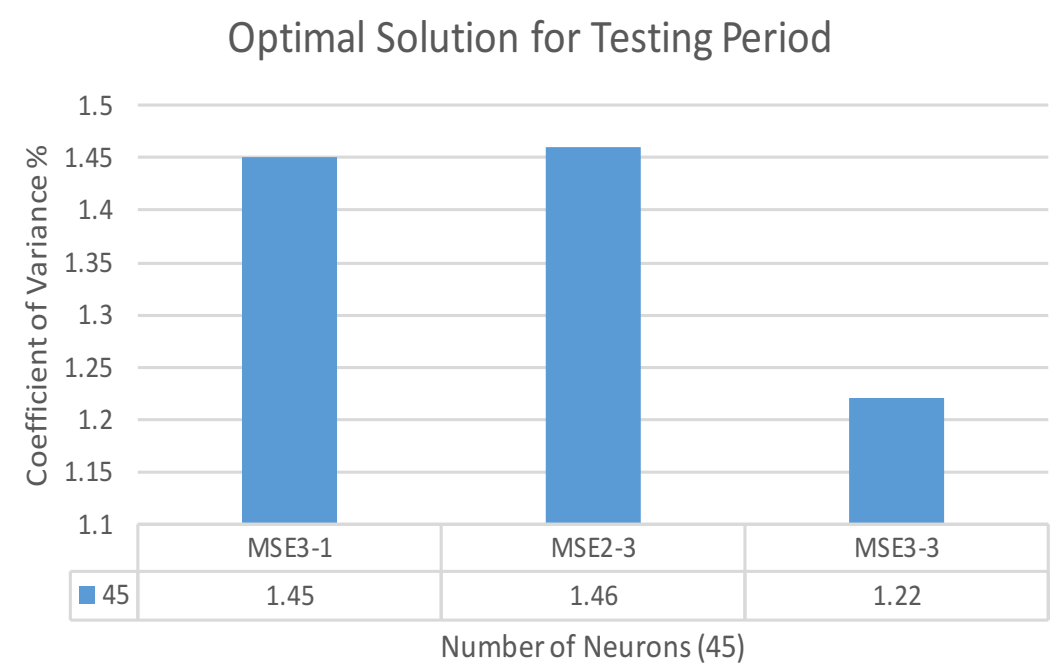

Fig. 10: The results for optimal model structure of the cooling coil model in terms of CV\%. (CV1-3 means a time delay of 1 and feedback delay of 3 )

\section{Optimal Solution for Testing Period}

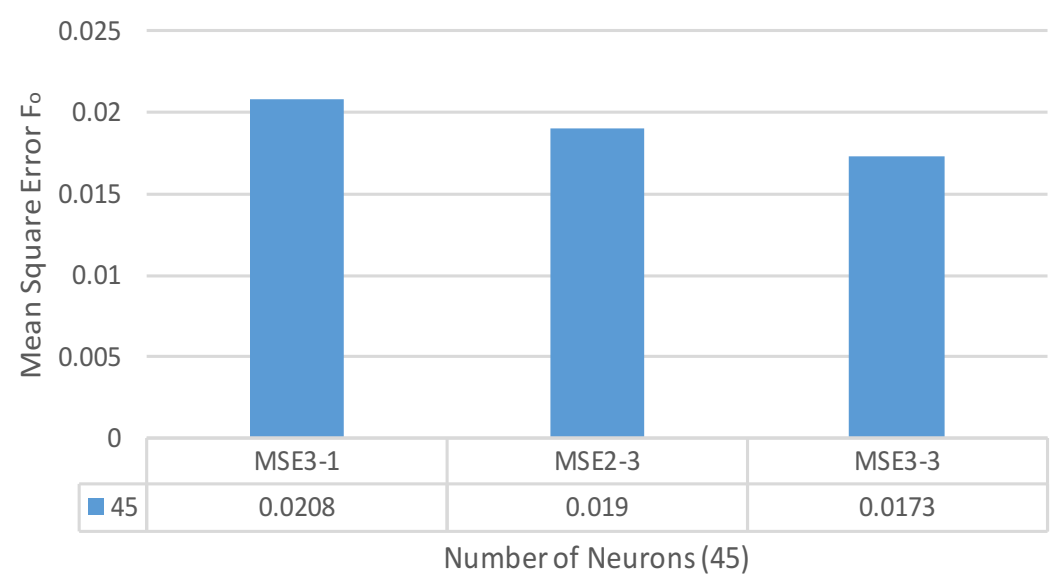

Fig. 11: The results for optimal model structure of the cooling coil model in terms of MSE. (MSE1-3 means a time delay of 1 and feedback delay of 3)

\section{Supply Fan Model Results}

A similar practice was applied for the fan model. the model was constructed to predict the fan power as a function of the fan air flow and fan speed. The actual data that were used in this simulation were actual data generated from a real building and are divided into two sets of data (1) is the training set, from November 1 st to December 31 st and (2) testing set was conducted from January 1st to February 1st. Results are tabulated in Table 1 and 2 and shown in Fig. 12 and 13.

\section{Optimization Process Results}

After performing a parametric study on the air handler unit, it is desirable to investigate the accuracy of the results by performing an optimization process. The genetic algorithm implemented was used to solve the objective function described previously in the methodology section. The genetic algorithm was limited to a maximum of 100 generations and a population size of 50. As seen in Table 1, the results for the minimum Coefficient of Variance (CV) are displayed for the cooling coil artificial neural network model. The moving average yielded the lowest CV with a value of 1.22. Additionally, the same model structure also held the lowest Mean Square Error (MSE) of 0.02. It is noted that the results for the time delay and order produced by the optimization tool are similar in value to those obtained in the parametric study. The optimization process as shown in Fig. 6 is applied. The resulted COVs and MSEs are also shown in 
Rand Talib et al. / American Journal of Engineering and Applied Sciences 2018, 11 (4): 1188.1198 DOI: 10.3844/ajeassp.2018.1188.1198

Table 1. The optimal model structure performance in the testing and training period is shown in Fig. 14 and 15.

Table 1: Optimal COVs and MSEs for the testing data set for cooling coil artificial neural network model

\begin{tabular}{lllll}
\hline Number of neurons & Time delay & Feedback delay & Minimum CV\% & Minimum MSE \\
\hline 45 & 1 & 3 & 1.45 & 0.019 \\
45 & 2 & 3 & 1.46 & 0.019 \\
45 & 3 & 3 & 1.22 & 0.020 \\
\hline
\end{tabular}

Table 2: Resulted optimal COVs and MSEs for the testing data set for the fan model

\begin{tabular}{llll}
\hline Number of neurons & Time delay & Minimum CV\% & Minimum MSE \\
\hline 30 & 1 & 18.63 & 0.555 \\
30 & 2 & 18.08 & 0.523 \\
30 & 3 & 18.15 & 0.527 \\
\hline
\end{tabular}

\section{Optimal solution for Testing period}

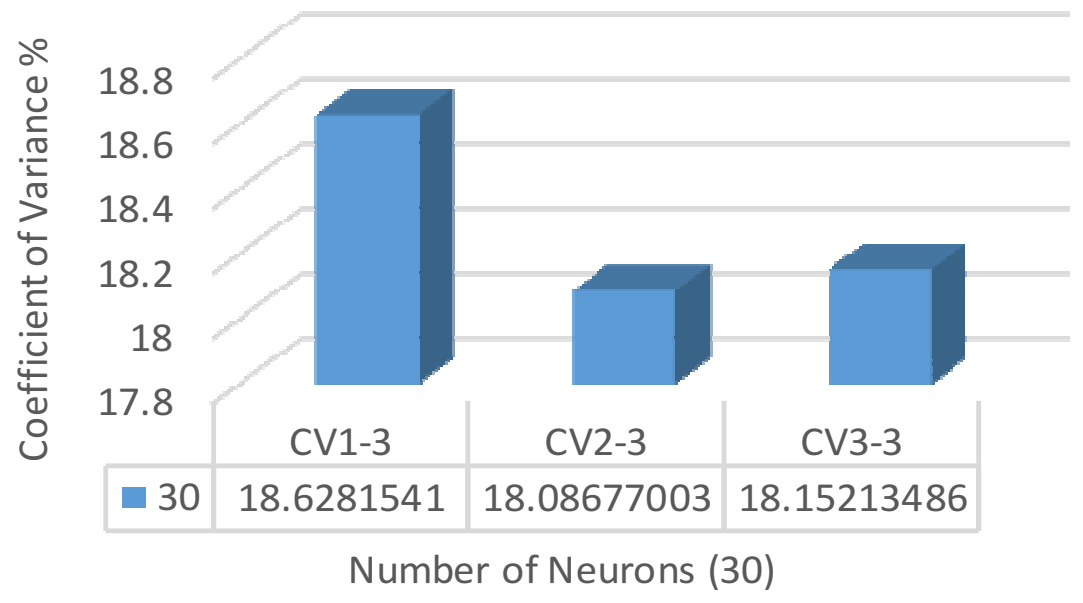

Fig. 12: The optimal model structure for the fan power model in terms of CV\%

\section{Optimal solution for Testing period}

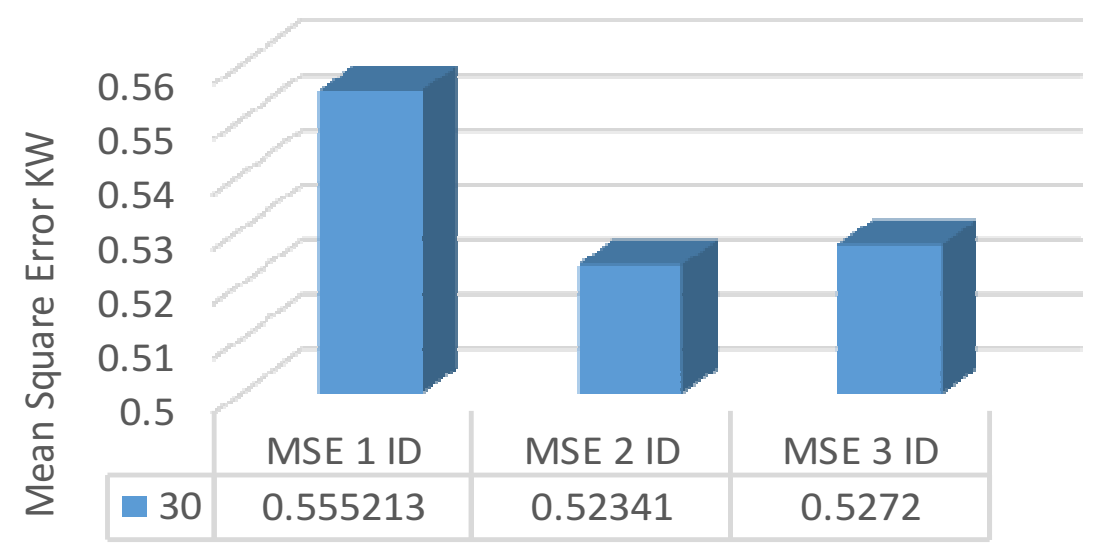

Number of Neurons (30)

Fig. 13: The optimal model structure for the fan power model in terms of MSE 


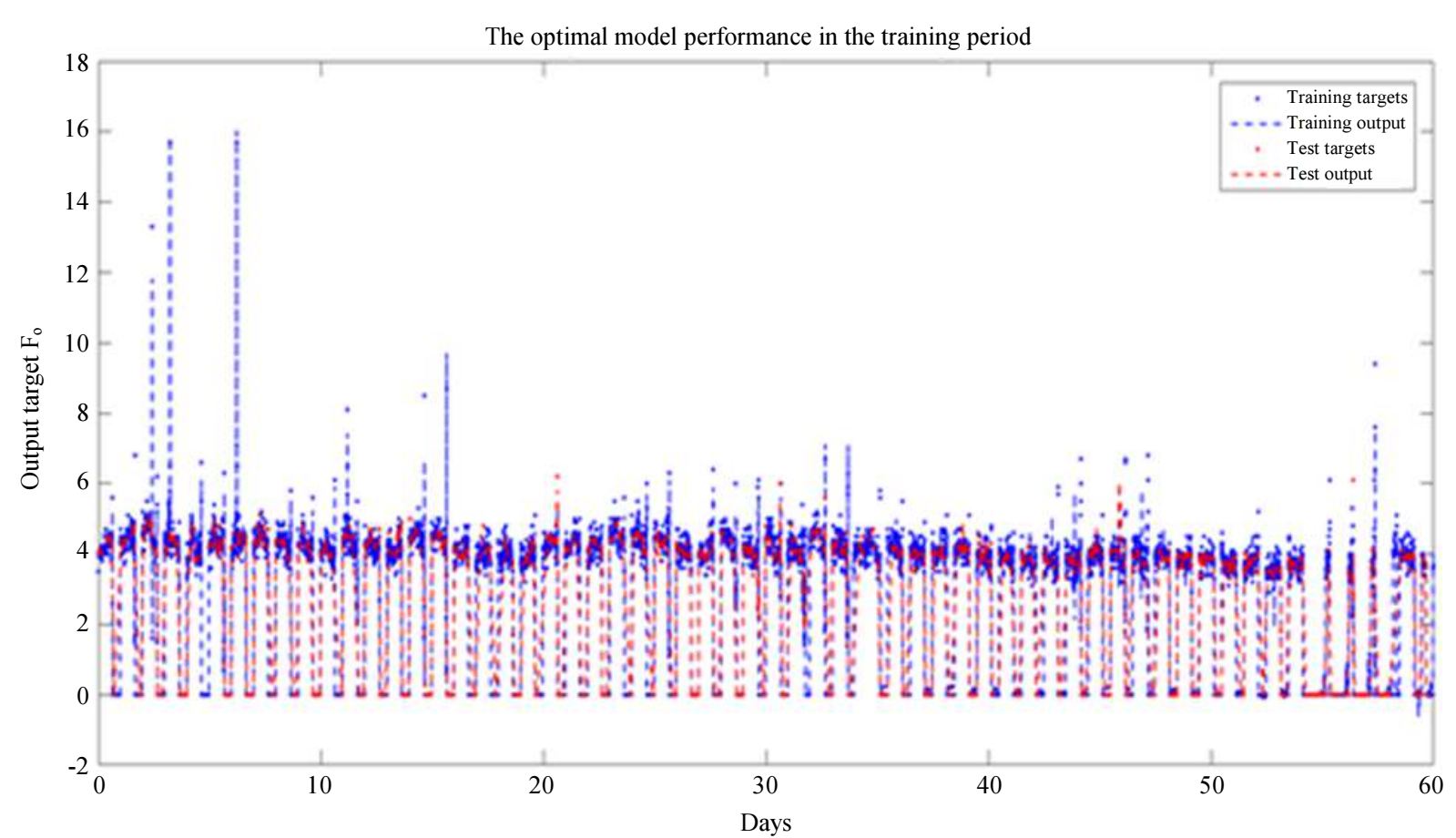

Fig. 14: The optimal model performance in the training period (training period is 60 days)

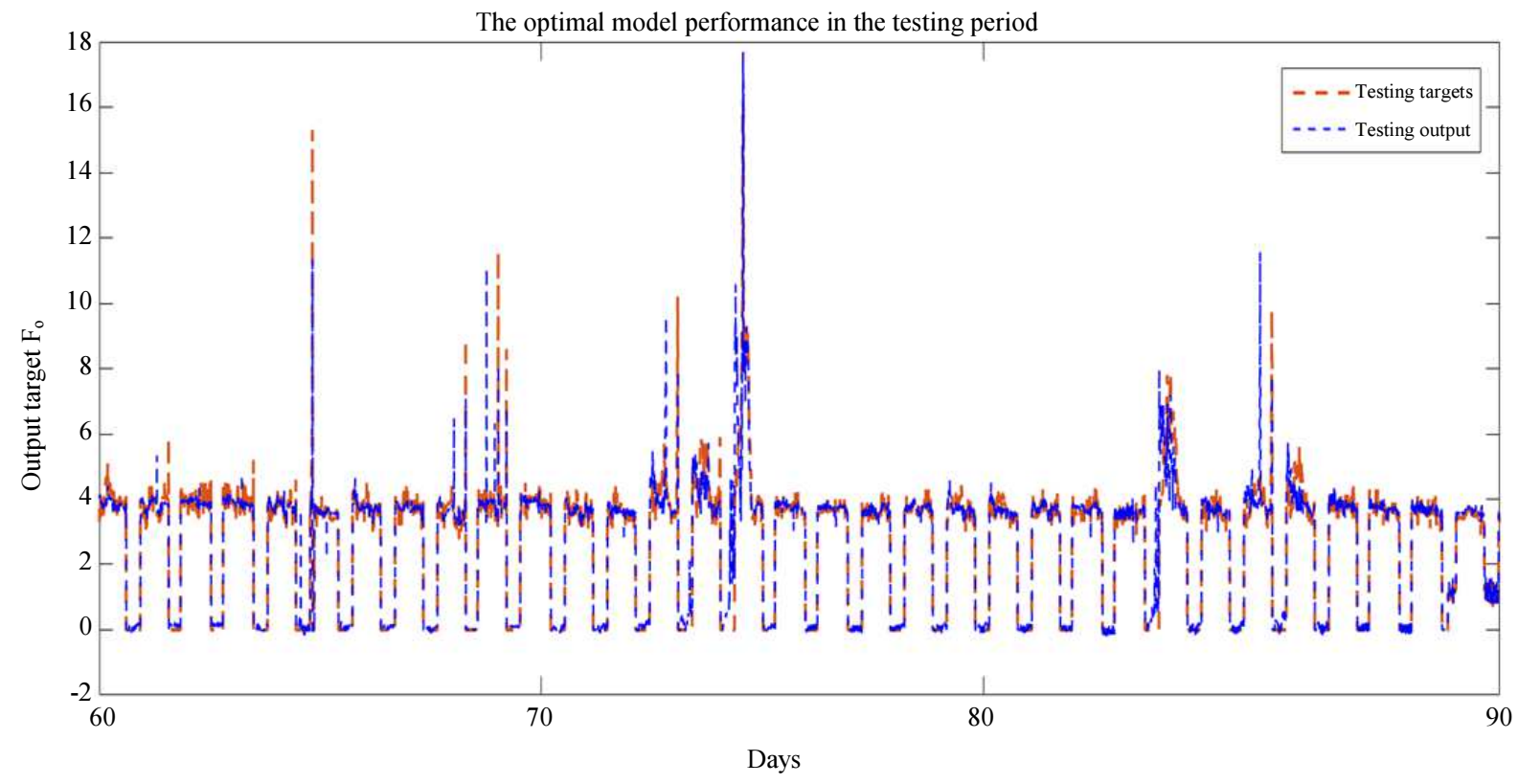

Fig. 15: The optimal model performance in the testing period (testing period is 30 days)

\section{Conclusion}

This research study was conducted to evaluate the effectiveness in applying Artificial Neural Networks to model the performance of cooling coil AHU. The results collected from this experiment has proved that the artificial neural networks can be used as a potential tool in predicting the performance of a chilled water air handling unit. New techniques are always being investigated to explore new intelligent methods to better understand the performance of HVAC systems. In this research the experiment was constructed using real time data collected from a building located in Greensboro, North Carolina and 
the ANN was chosen as the tool to model the performance of the collected data.

The integrated Building Automation System (BAS) software was the method of the data collection. Moreover, the CHW system was run at five-minute time intervals from November 2014 to February 2015. In total 28,767 sample points were collected from the system, representing more than 2,160 hours of system run time. Also, the network architectures were developed and tested using a every detailed combination of training algorithm, hidden layer neurons, sample data type and number of output variables. Additional testing was required when it was determined that the networks were being over-fitted, wherein a lower number of hidden neurons were used. After conducting the experiment using multiple input variables it was concluded that the networks with natural sample data with multiple output variables exhibited the best prediction performance in this experiment. The network's interfaced with the data much simpler than originally anticipated, requiring no more than 5 neurons to achieve peak performance in each configuration tested.

Two models were constructed to better understand the performance of the chilled water AHU. First, the cooling coil model which it results varied with the changing of the model variables that where selected for this research which are: (a) Feedback time delays (b) input time delays and (c) number of neurons (hidden layer size).

Second, the fan model which was tested by changing less variable than the cooling coil model. The error value in the fan model structure were higher than the cooling coil model. It was concluded that the higher error values were due to less number of inputs that were used in constructing the model. This can be avoided by increasing the number of inputs to get more accurate results and decrease the error value. Moreover, an optimization technique was developed using Genetic Algorithm (GA) to validate the results of the parametric study. The genetic algorithm was designed to have a maximum of 50 generations and a population size of 100

However, the optimal solution that was conducted through the optimization process was evaluated against the one generated by the parametric studies. The two introduced similar results were the model with the size of 40-50 number of neurons, time delay of 3 intervals and feedback delay of 3 intervals held the best results.

\section{Acknowledgement}

This work was supported by the U.S. Department of Commerce - NIST.

\section{Funding Information}

The work was financially supported by the U.S. Department of Commerce (NIST)/financial assistant award 70NANB14D1319.

\section{Author's Contributions}

All the authors contributed equally to this prepare, develop and carry out this manuscript.

\section{Ethics}

The authors declare that there are no ethical issues that could arise after the publication of this study.

\section{References}

ASHRAE, 2015. ASHRAE Handbook-Applications. 1st Edn., American Society of Heating Refrigeration and Air Conditioning Engineers, Inc, Atlanta.

ASHRAE, 2016. ASHRAE Handbook-Systems. 1st Edn., American Society of Heating Refrigeration and Air Conditioning Engineers, Inc., Atlanta.

Bishop, C.M., 1995. Neural Networks for Pattern Recognition. 1st Edn., Oxford University Press, New York, ISBN-10: 0198538642, pp: 482.

EIA, 2015. How much energy is consumed in residential and commercial buildings in the united states? U.S. Energy Information Administration - Independent Statistics and Analysis. Web.

Gallant, S.I., 1993. Neural Network Learning and Expert Systems. 1st Edn., MIT Press, Cambridge, ISBN-10: 0262071452 , pp: 365.

Goldberg, D.E., 1989. Genetic Algorithms in Search, Optimization and Machine Learning. 1st Edn., Addison-Wesley, Reading.

Nassif, N., 2014. Modeling and optimization of HVAC systems using artificial neural network and genetic algorithm. Build. Simulat., 7: 237-245. DOI: $10.1007 / \mathrm{s} 12273-013-0138-3$

Pérez-Lombard, L., J. Ortiz and C. Pout, 2008. A review on buildings energy consumption information. Energy Build., 40: 394-398. DOI: 10.1016/j.enbuild.2007.03.007 